\title{
Indicadores Sociais de Grávidas Adolescentes: Estudo Caso-Controle
}

\author{
Social Indicators of Pregnant Adolescents: A Case Control Study \\ Daniela Michelazzo ${ }^{1}$, Marta Edna Holanda Diógenes Yazlle ${ }^{1}$, Maria Célia Mendes ${ }^{1}$, \\ Maristela Carbol Patta ${ }^{1}$, Juan Stuardo Yazlle Rocha ${ }^{2}$, Marcos Dias de Moura ${ }^{1}$
}

\begin{abstract}
RESUM0
Objetivo: verificar diferenças entre alguns indicadores sociais de uma população de gestantes adolescentes (12 a 19 anos) e de adultas, procedentes de e que tiveram parto em Ribeirão Preto-SP, entre janeiro de 1992 e dezembro de 1996.

Métodos: foram analisadas informações relacionadas à internação, obtidas das folhas de alta hospitalar no Centro de Processamento de Dados Hospitalares do Departamento de Medicina Social, FMRP-USP. Os parâmetros analisados foram: tipo e número de partos, categoria de internação, ocupação e diagnósticos obstétricos. Para processar as informações, foram utilizados o Sistema Epi-Info processador de texto 6.04a, banco de dados e estatística para epidemiologia, produzido pelos Centers of Disease Control and Prevention (Atlanta, GA, USA), e o Dbase IV. A associação entre variáveis foi testada pelo $\chi^{2}$ com nível de significância de 5\%, usando o software GraphPad Prism versão 2.0, 1995.

Resultados: ocorreram 43.253 partos no periodo, sendo 7.134 (16,5\%) de adolescentes $e$ $36.119(83,5 \%)$ de adultas. Observou-se aumento de $25,5 \%$ no número de partos nas adolescentes no decorrer dos anos. A proporção de partos de adolescentes aumentou significativamente na categoria SUS no período. A proporção de internações de adolescentes pelo SUS foi significativamente superior à de adultas. Apenas 14,1\% das adolescentes tinham inserção na população economicamente ativa, comparado com $34,8 \%$ das adultas. Apenas $6,8 \%$ das adolescentes eram estudantes, ao passo que 79,0\% eram "do lar" ou sem ocupação remunerada. Houve aumento da proporção de parto vaginal entre adolescentes quando comparadas às mulheres adultas, enquanto a proporção de cesáreas permaneceu estável e maior entre as adultas. O trabalho de parto prematuro ou falso foi significativamente mais freqüente entre as adolescentes.

Conclusões: observamos aumento do número de partos entre adolescentes, sendo a maioria normal. Tanto a proporção de partos pelo SUS quanto a proporção de partos vaginais foi maior entre a população de adolescentes. Houve predomínio de adolescentes com atividades no lar e sem remuneração. Assim, recomendamos medidas para prevenção de gestação na adolescência, com ênfase à população mais carente.
\end{abstract}

PALAVRAS-CHAVE: Adolescência. Resolução da gravidez. Complicações da gravidez. Cesariana. Parto normal.

${ }^{1}$ Departamento de Ginecologia e Obstetrícia da Faculdade de Medicina de Ribeirão Preto, Universidade de São Paulo, Brasil. ${ }^{2}$ Departamento de Medicina Social da Faculdade de Medicina de Ribeirão Preto da Universidade de São Paulo. Correspondência:

Marta Edna Holanda Diógenes Yazlle

Departamento de Ginecologia e Obstetrícia

Faculdade de Medicina de Ribeirão Preto, USP

Av. Bandeirantes $3900-8^{\circ}$ andar - Campus Universitário

14049-900 - Ribeirão Preto - SP

Telefone: (16) 602-0216 - Fax: (16) 602-0946

\section{Introdução}

A gravidez na adolescência é considerada, em alguns países, um problema de saúde pública. Alguns estudos fazem referências aos efeitos negativos que a gestação, nesta faixa etária, pode acarretar à saúde da mulher e à sua inserção no 
mercado de trabalho, refletindo no seu crescimento pessoal e profissional ${ }^{1-3}$.

No que diz respeito à evolução da gestação, a literatura cita entre as adolescentes: aumento da incidência de prematuridade ${ }^{4,5}$, baixo peso ao nascimento ${ }^{4}$, restrição de crescimento intrauterino, sofrimento fetal agudo intraparto, diabete gestacional, pré-eclâmpsia e aumento da incidência de cesarianas ${ }^{1,6-8}$. Por outro lado, alguns estudos mostram que não há prejuízo na evolução da gestação e condição dos recém-nascidos quando a gestação ocorre na adolescência, desde que a assistência pré-natal seja adequada ${ }^{2,9-11}$. Além do mais, a literatura sugere que as condições sociais em que as gestantes estão inseridas, especialmente as adolescentes, podem interferir decisivamente na evolução da gravide $z^{12}$.

O estado gravídico e a maternidade na adolescência exercem efeitos negativos sobre a qualidade de vida, uma vez que prejudicam as condições de estudo e intensificam as dependências familiares, advindo, assim, conseqüências desfavoráveis na perspectiva de vida e trabalho ${ }^{13}$.

Com base nas informações da literatura, propusemos a realizar o presente estudo, com o objetivo de averiguar se essa problemática ocorre em nosso meio, ou seja, se o aumento do número de partos entre adolescentes é real se a evolução da gestação nas adolescentes é diferente de mulheres adultas e se existe interferência de alguns indicadores sociais entre número e tipo de partos entre adolescentes e adultas.

\section{Pacientes e Métodos}

Utilizando o sistema de informações de altas hospitalares do Centro de Processamento de Dados Hospitalares (CPDH) do Departamento de Medicina Social da FMRP-USP, os autores avaliaram, retrospectivamente, os dados referentes aos partos de mulheres procedentes de Ribeirão Preto, São Paulo, na faixa etária de 11 a 19 anos e adultas, que ocorreram no município de Ribeirão Preto, no período de janeiro de 1992 a dezembro de 1996.

Para processar as informações, foram utilizados: o programa Epi-Info processador de texto $6.04 a$, banco de dados e estatística para epidemiologia, produzido pelos Centers of Disease Control and Prevention (Atlanta, GA, USA) e o Dbase IV. A associação entre as variáveis foi testada pelo $\chi^{2}$ com nível de significância de $5 \%$, usando o software GraphPad Prism versão 2.0, 1995.

Como indicadores sociais foram usados a categoria de internação e o tipo de ocupação des- sas pacientes. A categoria de internação foi classificada segundo o seu financiamento em: particular, pré-pagamento e sistema único de saúde (SUS), que representam a posição social das pacientes, de acordo com o trabalho de Rocha et al. ${ }^{14}$.

As ocupações das mulheres internadas foram codificadas segundo a Classificação Brasileira de Ocupações para o subsistema de informações sobre mortalidade (Ministério da Saúde, 1987). Estas ocupações são agrupadas em estratos sociais, segundo o modelo proposto pelo Registrar General na Inglaterra ${ }^{15}$, levando em consideração alguns critérios utilizados por Singer et al. ${ }^{16}$, na Bahia. Assim, as mulheres foram distribuídas entre população economicamente ativa (PEA) e fora da PEA. A classificação fora da PEA corresponde às ocupações tais como: donas de casa, estudantes, menores, aposentados e desempregados. A classificação PEA corresponde, segundo o tipo de trabalho, a: profissional, intermediário, qualificado não manual, qualificado manual, semiqualificado e não qualificado. Segundo Rocha et al. ${ }^{14}$, esta estratificação representa a posição socioeconômica de importância decrescente.

Para avaliação dos diagnósticos obstétricos, foram utilizadas as informações referidas nas folhas de altas hospitalares, de acordo com a Classificação Internacional de Doenças (CID), $9^{\text {a }}$ revisão $(1975)^{17}$. Os diagnósticos obstétricos mais freqüentes associados ao parto foram: problemas do feto ou placenta que afetam a conduta materna, como malformação fetal hereditária ou causada por vírus ou drogas, hemorragia feto-materna, isoimunização $\mathrm{Rh}$ ou $\mathrm{ABO}$, sofrimento fetal, morte intra-uterina, crescimento fetal insuficiente ou excessivo, anormalidade placentária, desproporção feto-pélvica, problemas com a cavidade amniótica e membranas, hipertensão complicando o parto e o puerpério e trabalho de parto prematuro ou falso. $\mathrm{O}$ trabalho foi analisado pelo Comitê de Ética em Pesquisa do Hospital das Clínicas da Faculdade de Medicina de Ribeirão Preto da Universidade de São Paulo em sua $151^{\text {a }}$ Reunião Ordinária e aprovado de acordo com o Processo HCRP n ${ }^{\circ}$ 1822/2003.

\section{Resultados}

Ocorreram, no período referido, 43.253 partos em Ribeirão Preto de mulheres provenientes deste município, sendo que 7.134 foram de adolescentes, correspondendo a $16,5 \%$ do total de partos, ao passo que $36.119(83,5 \%)$ foram de mulheres adultas.

Observou-se aumento do número de partos 
de adolescentes no decorrer dos anos, tendo ocorrido 1.225 partos em 1992 e 1.538 em 1996. O número de partos de mulheres adultas se manteve estável, embora tenham ocorrido pequenas oscilações, ou seja: 7.002 partos em 1992, 7.134 partos em 1993, 7.445 partos em 1994, 7.478 em 1995 e 7.060 em 1996 (Figura 1). Houve diferença significante entre a proporção de partos de adolescentes e adultas no decorrer dos anos, a saber: em 1992: 14,9\% vs 85,1\%; em 1993: $16,3 \%$ vs 83,7\%; em 1994: 16, $1 \%$ vs $83,9 \%$; em 1995: $17,2 \%$ vs $82,8 \%$ e em 1996: $17,9 \%$ vs $82,1 \%$, respectivamente $(\mathrm{p}<0,0001)$.

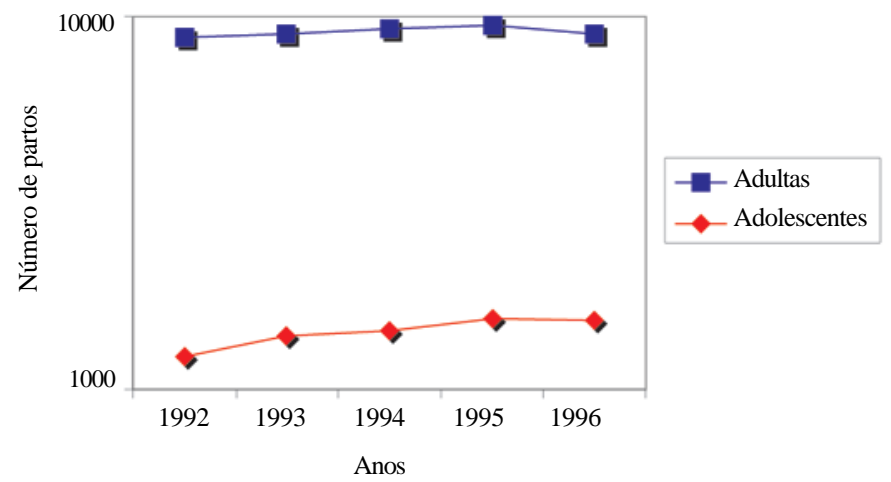

Figura 1 - Distribuição dos partos em adolescentes e adultas em Ribeirão Preto-SP, no período de 1992-1996.

Na Tabela 1 encontra-se a freqüência dos partos segundo a idade materna. Observam-se partos em adolescentes a partir dos 12 anos, havendo elevação gradual deste número com o aumento da idade. Comparando o número de partos em 1996 com os partos de 1992, em cada grupo etário a partir dos 14 anos, observamos crescimento de $104,2 \%$ para os partos nesta idade, $48,8 \%$ aos 15 anos, $36,1 \%$ aos 16 anos, $14 \%$ aos 17 anos, $52,8 \%$ aos 18 anos e, praticamente, não houve aumento entre as mães com 19 anos de idade.

Com o aumento da idade, o número de partos foi crescente, variando de $0,06 \%$ aos 12 anos a $29,1 \%$ aos 19 anos, destacando-se que dos 15 aos 16 anos houve aumento de 7,1\% a 14,2\% . Nas adultas a maioria dos partos se deu entre os 20 e os 30 anos $(73,2 \%)$.

Quanto à categoria de internação, observouse que a proporção de partos entre a população de adolescentes aumentou na categoria SUS, em relação às mulheres adultas, com o decorrer dos anos no período de 1992 a 1996. Em 1992, 19,3\% dos partos do SUS foram em adolescentes, ao passo que em 1996 essa proporção aumentou para 24,6\%. Nas categorias particular e pré-pagamento não houve diferença na proporção de partos entre adolescen- tes e adultas. A evolução da proporção de partos entre a população de adolescentes em cada categoria de internação é mostrada na Figura 2.

Tabela 1 - Freqüência de partos em Ribeirão Preto-SP, de acordo com a idade materna, no período de 1992-1996.

\begin{tabular}{crrrrr}
\hline Idade em anos & 1992 & 1993 & 1994 & 1995 & 1996 \\
\hline 12 & 0 & 1 & 2 & 1 & 0 \\
13 & 5 & 3 & 6 & 8 & 8 \\
14 & 24 & 30 & 41 & 36 & 49 \\
15 & 82 & 88 & 93 & 122 & 122 \\
16 & 169 & 191 & 192 & 219 & 230 \\
17 & 257 & 256 & 269 & 321 & 293 \\
18 & 284 & 426 & 388 & 408 & 434 \\
19 & 404 & 394 & 440 & 436 & 402 \\
Total & 1225 & 1389 & 1431 & 1551 & 1538 \\
20 ou + & 7002 & 7134 & 7445 & 7478 & 7050 \\
\hline
\end{tabular}

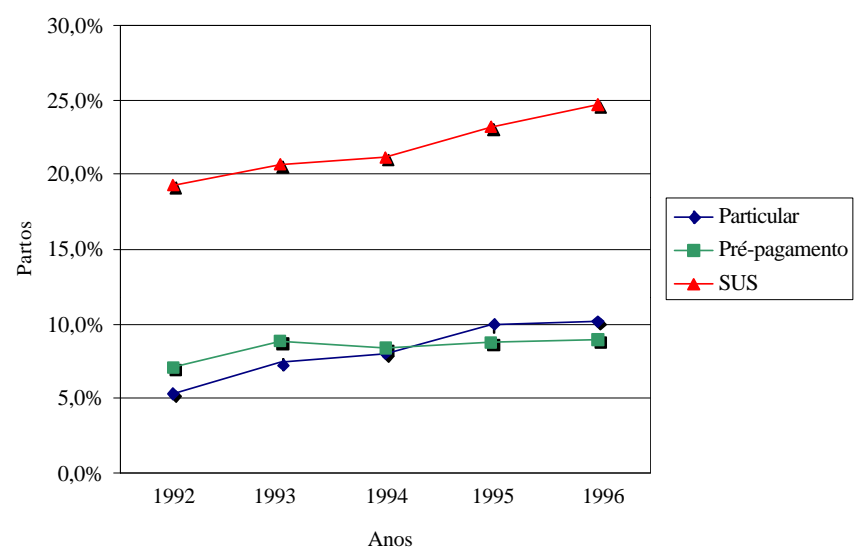

Figura 2 - Distribuição da proporção dos partos de adolescentes em relação à de adultas, de acordo com a categoria de internação, em Ribeirão Preto, SP, no período de 1992-1996.

Na Tabela 2 está distribuída a freqüência de partos na população de adolescentes e adultas de acordo com a categoria de internação. A proporção de adolescentes internadas pelo SUS foi significativamente superior à de adultas na mesma categoria ( $\mathrm{p}<0,0001)$. No SUS ocorreram partos em adolescentes a partir de 12 anos, ao passo que nas categorias pré-pagamento e particular, a idade mínima das gestantes foi de 13 e 14 anos, respectivamente.

No que se refere à ocupação, 1.005 (14,1\%) adolescentes grávidas já tinham inserção na PEA, 482 (6,8\%) eram estudantes e 5.637 (79,0\%) eram do lar, menores e sem ocupação remunerada. Para dez pacientes $(0,1 \%)$ não havia informação sobre a ocupação. Dentre a população adulta, 12.556 (34,8\%) 
já tinham inserção na PEA, $343(0,9 \%)$ eram estudantes e $23.100(64,3 \%)$ eram do lar e sem ocupação remunerada. Para 20 pacientes $(0,1 \%)$ não havia informação sobre a ocupação.

Tabela 2 - Distribuição do total de partos de mulheres adolescentes e adultas em Ribeirão Preto-SP, no período de 1992-1996, segundo a categoria de internação.

\begin{tabular}{|c|c|c|c|c|c|c|c|}
\hline & \multicolumn{2}{|c|}{ Particular } & \multicolumn{2}{|c|}{ Pré-pagament } & \multicolumn{2}{|c|}{ SUS } & \multirow[t]{2}{*}{ Tota } \\
\hline & $\mathrm{n}$ & $\%$ & $\mathrm{n}$ & $\%$ & $\mathrm{n}$ & $\%$ & \\
\hline Adolescentes & 148 & 2,1 & 1277 & 17,9 & 5709 & 80,0 & \\
\hline Adultas & 1692 & 4,7 & 13828 & 38,3 & 20599 & 57,0 & 361 \\
\hline
\end{tabular}

Com relação ao tipo de parto no decorrer do período avaliado, houve aumento da proporção de parto vaginal (normal e fórceps) entre as adolescentes quando comparadas às mulheres adultas. Em 1992, 18,9\% dos partos normais e 29,1\% dos partos fórceps foram em adolescentes, ao passo que em 1996 essa proporção aumentou para $22,7 \%$ e $48,0 \%$, respectivamente. A proporção de cesáreas não apresentou alteração estatisticamente significativa no decorrer dos anos. A evolução da proporção dos tipos de parto entre a população de adolescentes em relação à de adultas é mostrada na Figura 3.

Ainda com relação ao tipo de parto, a proporção global de parto normal foi de $59,2 \%$ entre as adolescentes e $44,8 \%$ entre as adultas e a de fórceps foi 5,6\% entre as adolescentes e 1,6\% entre as adultas. A proporção de cesariana foi de $35,2 \%$ entre as adolescentes e 53,6\% entre as adultas. Todas as diferenças foram estatisticamente significativas $(\mathrm{p}<0,001)$.

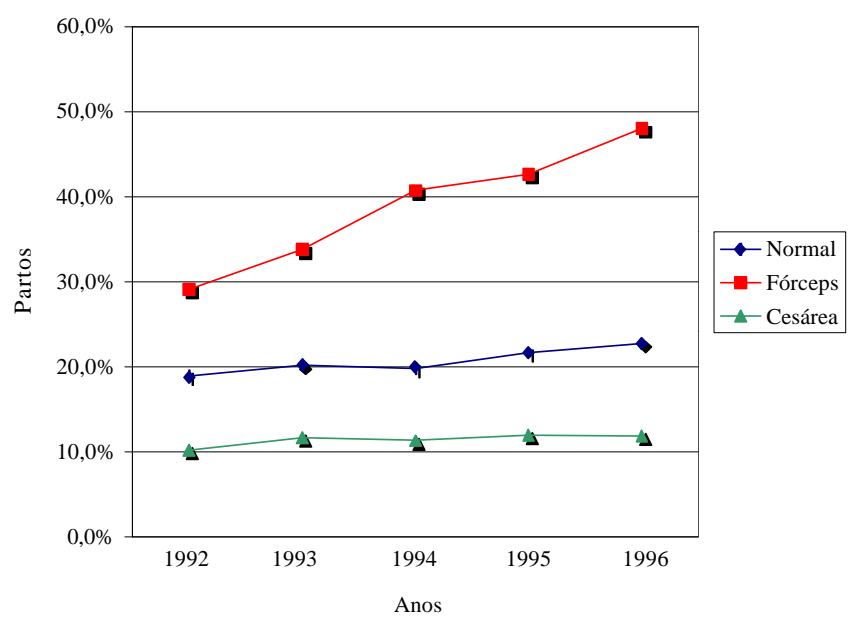

Figura 3 - Distribuição da proporção dos tipos de parto entre a população de adolescentes em relação à de adultas, em Ribeirão Preto, SP, no período de 1992-1996.

Quanto aos diagnósticos obstétricos mais freqüentes associados ao parto, de acordo com a CID ( $9^{\text {a }}$ revisão de 1975) ${ }^{17}$, observamos: problemas do feto ou placenta que afetam a conduta materna, como malformação fetal hereditária ou causada por vírus ou drogas, cuja proporção de ocorrência foi maior na população adulta $(10,1 \%$ vs 7,9\%); desproporção feto-pélvica e problemas com a cavidade amniótica e membranas, ambos sem diferença estatisticamente significativa entre as populações de adolescentes e adultas $(6,0 \%$ vs $6,2 \%$ e 5,0 vs 5,1\%, respectivamente); hipertensão complicando o parto e o puerpério, também com maior proporção entre as adultas $(4,5 \%$ vs $3,5 \%)$, e, finalmente, trabalho de parto prematuro ou falso, que ocorreu em maior proporção na população de adolescentes $(3,4 \%$ vs $1,6 \%)$ (Tabela 3$)$.

Tabela 3 - Distribuição dos diagnósticos obstétricos mais freqüentes associados ao parto de adolescentes e adultas em Ribeirão Preto-SP, no período de $1992-1996$.

\begin{tabular}{|c|c|c|c|c|c|}
\hline & \multicolumn{2}{|c|}{$\begin{array}{c}\text { Adolescentes } \\
(n=7134)\end{array}$} & \multicolumn{2}{|c|}{$\begin{array}{l}\text { Adultas } \\
(n=36119)\end{array}$} & \multirow[t]{2}{*}{ p } \\
\hline & n & $\%$ & $\mathbf{n}$ & $\%$ & \\
\hline Problemas fetais ou da placenta afetando a conduta em relação à mãe (656) & 565 & 7,9 & 3633 & 10,1 & $<0,01$ \\
\hline Desproporção feto-pélvica (653) & 426 & 6,0 & 2252 & 6,2 & 0,4 \\
\hline Problemas com a cavidade amniótica e membranas (658) & 355 & 5,0 & 1854 & 5,1 & 0,6 \\
\hline Hipertensão complicando o parto e puerpério (642) & 249 & 3,5 & 1623 & 4,5 & $<0,01$ \\
\hline Trabalho de parto prematuro ou falso (644) & 241 & 3,4 & 576 & 1,6 & $<0,01$ \\
\hline
\end{tabular}

\section{Discussão}

Para realizar este estudo foram obtidos dados a partir de bases populacionais, ou seja, foram in- cluídos todos os hospitais e as hospitalizações da cidade e região de Ribeirão Preto, onde a assistência ao parto é totalmente assegurada, ou seja, onde todas as pacientes têm garantida assistência hospitalar ao parto ${ }^{1}$. A proporção de partos entre ado- 
lescentes em relação ao total de partos, no período de janeiro de 1992 a dezembro de 1996, foi de 16,5\%. Houve crescimento do número de partos entre a população de adolescentes com o decorrer do tempo, de 1992 para 1996, ao passo que o número de partos entre a população adulta permaneceu estável. No Brasil, Prado ${ }^{18}$ referiu incidência de partos em adolescentes correspondente a $21 \%$, e Costa et al. ${ }^{19}$, a $21,6 \%$. Nos Estados Unidos, segundo Martin et al. ${ }^{20}$, houve redução das taxas de nascimento na população de adolescentes a partir de 1991. Segundo a idade das adolescentes, de 1992 para 1996, observamos maior aumento percentual aos 14 anos $(104,2 \%)$, diminuindo gradualmente até os 17 anos (14\%). Aos 18 anos houve nova elevação $(52,8 \%)$, que pode ser explicada pela iniciação sexual precoce no nosso meio.

É ampla a discussão sobre a relação entre gravidez na adolescência e nível socioeconômico. Guijarro et al..$^{21}$, estudando adolescentes grávidas, relatou comunicação intrafamiliar deficiente, baixa satisfação pessoal e felicidade, além de mais dificuldades escolares e econômicas nesta população. Outros autores mostram que as adolescentes que não engravidam apresentam mais religiosidade, pais mais educados e os pais trabalhando fora de casa ${ }^{14,22}$.

De acordo com Lesser e Escoto-Lloyd ${ }^{3}$, outro importante fator que contribui para os problemas de saúde das adolescentes grávidas e das mães jovens é a falta de recursos sociais. Com a maternidade, há modificação radical no estilo de vida das adolescentes, que acabam sobrecarregadas ao tentar equilibrar estudo, trabalho e a administração de um novo lar. Além disso, não podemos ignorar a discriminação, que ainda hoje existe, tanto por parte da sociedade como da própria família, o que pode determinar sérias conseqüências de ordem psicológica e social.

No presente estudo, observamos maior porcentagem dos partos, tanto de adolescentes quanto de adultas, na categoria de internação SUS, quando comparado às outras categorias (pré-pagamento e particular), o que está de acordo com o que acontece com a população geral de mulheres no município de Ribeirão Preto ${ }^{22}$. Também observamos, no decorrer dos anos, elevação da proporção de partos de adolescentes na categoria de internação SUS em relação à proporção de partos de adultas. Isto sugere que as adolescentes são de nível socioeconômico baixo, devendo ser adotadas algumas medidas de prevenção da gravidez na adolescência, principalmente dirigidas às adolescentes de nível socioeconômico baixo, o que corresponde, neste município, à população atendida pelo SUS ${ }^{12}$.
É alta a taxa de evasão escolar entre as adolescentes grávidas, aproximando-se dos $30 \%$. Infelizmente, na maioria dos casos, o retorno ao estudo se dá em menores proporções ${ }^{23,24}$. Entretanto, muitas vezes o abandono escolar ocorre antes da gravidez, sendo inclusive fator de risco para engravidar ${ }^{13}$. Em vista disso, adolescentes que abandonam seus estudos não se profissionalizam e, conseqüentemente, terão trabalho mal remunerado no futuro.

Neste estudo, 85\% das adolescentes foram classificadas como fora da PEA, portanto não tinham trabalho remunerado, sendo a maioria pertencente à categoria do lar. A idade é um dos fatores que determina a exclusão destas pacientes da PEA, pois nesta fase da vida ainda não se possui capacitação profissional $^{22}$. Com a ocorrência de gravidez neste período, a chance de entrar para a PEA torna-se ainda menor. Se considerarmos que as pacientes pertencentes à categoria SUS são de nível socioeconômico mais baixo, confirmam-se assim os dados da literatura, o que leva à possibilidade de que permaneçam em categoria social menos favorecida.

Com relação ao tipo de parto, observou-se que, apesar de ter havido aumento na proporção de partos vaginais entre as adolescentes, a taxa de cesarianas ainda permanece elevada, tendo correspondido a $35,2 \%$ do total daqueles partos. A proporção de cesariana entre as adultas foi ainda maior, de $53,6 \%$, compatível com a elevada taxa nacional de cesárea mostrada pela literatura na população adulta ${ }^{22,25,26}$. Estes índices refletem-se na população mais jovem, estando provavelmente relacionados aos fatores que levaram à mudança na prática obstétrica no Brasil, principalmente entre as pacientes atendidas via categorias prépagamento e particular. O menor índice de cesariana entre as adolescentes do que entre as adultas pode ser explicado pela maior proporção de adolescentes atendidas pelo SUS, que em grande parte é representado em Ribeirão Preto pelo atendimento da Faculdade de Medicina de Ribeirão Preto da Universidade de São Paulo, que tenta manter as indicações de cesariana dentro das preconizadas pela literatura mundial.

Outro fator que também poderia justificar as taxas elevadas de cesárea no estudo em questão é a presença de um expressivo número de intercorrências obstétricas, como o parto pré-termo, as sindromes hipertensivas e a desproporção feto-pélvica. São intercorrências da gravidez semelhantes àquelas observadas na literatura ${ }^{6,27}$.

Existe controvérsia quanto ao fato de a gravidez na adolescência aumentar o risco de complicações obstétricas, perinatais e neonatais. Al- 
guns autores julgam que há elevação do risco ${ }^{8,27,28}$, ao passo que outros acreditam que o risco dessas complicações não aumenta na adolescente, quando comparado à população adulta ${ }^{2,9-11}$. Em nosso estudo, observamos maior incidência de trabalho de parto prematuro ou falso entre as adolescentes, ao passo que a incidência das demais doenças foi ou maior entre as adultas ou igual entre os grupos.

A maior incidência de parto prematuro entre as adolescentes encontrada no presente estudo está de acordo com o artigo de Balaka et al. ${ }^{29}$, segundo o qual adolescentes solteiras e com baixo nível educacional e socioeconômico são grupo de risco para parto pré-termo. Wasunna e Mohammed $^{28}$ também encontraram maior incidência de parto prematuro entre mães adolescentes, sendo este um grupo que merece cuidados especiais durante o pré-natal, parto e puerpério, na prevenção desta patologia.

Os resultados apresentados permitem concluir que: houve aumento da proporção de partos entre adolescentes no município de Ribeirão Preto de janeiro de 1992 a dezembro de 1996, enquanto a proporção de partos na população adulta permaneceu inalterada; o maior aumento se deu na faixa etária de 14 a 18 anos; nos dois grupos a maioria dos partos foi normal, ocorrendo aumento anual da proporção de partos vaginais, tanto normais quanto fórceps, entre as adolescentes, enquanto a proporção de cesáreas permaneceu estável; a maioria das adolescentes internadas pertencia à categoria SUS; houve aumento significativo dos partos de adolescentes na categoria SUS quando comparado com os partos de mulheres adultas na mesma categoria de internação; houve predomínio de adolescentes sem ocupação remunerada; dentre as doenças obstétricas referidas, foi significativamente mais freqüente, entre as adolescentes, o trabalho de parto prematuro ou falso (CID 644).

Pelo exposto sobre o assunto e considerando os resultados encontrados no presente estudo, pensamos que medidas estratégicas para prevenção da gestação na adolescência devem ser direcionadas àquelas pertencentes aos diferentes niveis socioeconômicos, com ênfase à população mais carente, representada pela categoria SUS.

\section{ABSTRACT}

Purpose: to check whether there were differences in some social indicators between adolescent and adult pregnant women in the city of Ribeirão Preto, from January 1992 to
December 1996

Methods: the information was obtained from hospital discharge forms and was analyzed at the Hospital Data Processing Center of the FMRP-USP. The analyzed parameters were: number and types of deliveries, category of hospital admission, occupation, and obstetric diagnosis. The 6.04a text processor Epi-Info System, a data bank and statistics of epidemiology produced by the Centers of Disease Control and Prevention (Atlanta, GA, USA), and Dbase IV were used to process the information. The association between variables was tested by the $\chi^{2}$ test, with level of significance set at $5 \%$, using the GraphPad Prism version 2.0, 1995 software.

Results: a total of 43,253 deliveries occurred during this period, among which 7,134 (16.5\%) corresponded to adolescent deliveries, while 36,119 (83.5\%) to adult deliveries. The number of deliveries by adolescent girls increased $25.5 \%$ along this period. The proportion of adolescent deliveries in the unified health system category of admission increased, and it was higher than that of the adults'. Only $14.1 \%$ of the adolescents belonged to the economically active population, comparing with $34.8 \%$ of the adults. Only $6.8 \%$ of the adolescents were students, while $79.0 \%$ were house-workers or had a nonpaid occupation. In the analyzed period, the ratio of vaginal delivery increased among the adolescents, as compared to that of the adults. The ratio of cesarean delivery persisted stable and higher among the adults. Premature delivery and false labor were significantly more frequent among the adolescents.

Conclusions: the number of deliveries increased among the adolescents, and most of them were normal. The ratio of admission by the unified health system category and that of vaginal delivery were higher among the adolescents. There were more adolescents without an economically active work. Thus, we recommend strategies to prevent adolescent pregnancy, mainly among the poor population.

KEYWORDS: Adolescence. Resolution of pregnancy. Pregnancy complications. Cesarean section. Normal delivery.

\section{Referências}

1. Barros FC, Vaughan JP, Victora CG, Huttly SR. Epidemic of caesarean sections in Brazil. Lancet 1991; 338:167-9.

2. Lao TT, Ho LF. The obstetric implications of teenage pregnancy. Hum Reprod 1997; 12:2303-5.

3. Lesser J, Escoto-Lloyd S. Health-related problems in a vulnerable population: pregnant teens and adolescent mothers. Nurs Clin North Am 1999; 14:289-99.

4. Fraser AM, Brockert JE, Ward RH. Association of young maternal age with adverse reproductive outcomes. N Engl J Med 1995; 332:1113-7. 
5. Jolly MC, Sebire N, Harris J, Robinson S, Regan L. Obstetric risks of pregnancy in women less than 18 years old. Obstet Gynecol 2000; 96:962-6.

6. Orvos H, Nyirati I, Hajdu J, Pal A, Nyari T, Kovacs L. Is adolescent pregnancy associated with adverse perinatal outcome? J Perinat Med 1999; 27:199-203.

7. Abu-Heija A, Ali AM, Al-Dakheil S. Obstetrics and perinatal outcome of adolescent nulliparous pregnant women. Gynecol Obstet Invest 2002; 53:90-2.

8. Eure CR, Lindsay MK, Graves WL. Risk of adverse pregnancy outcomes in young adolescent parturients in an inner-city hospital. Am J Obstet Gynecol 2002; 186:918-20.

9. Berenson AB, Wiemann CM, McCombs SL. Adverse perinatal outcomes in young adolescents. J Reprod Med 1997; 42:559-64.

10.Valdez-Banda F, Valle-Virgen O. Prevalence of risk factors for obstetric complications in adolescents. Comparison with the adult population. Ginecol Obstet Mex 1996; 64:209-13.

11.Weerasekera DS. Adolescent pregnancies - is the outcome different? Ceylon Med J 1997; 42:16-7.

12.Rocha JSY, Simões BJG. Estudo da assistência hospitalar pública e privada em bases populacionais, 1986-1996. Rev Saúde Pública 1999; 33:44-54.

13.Stevens-Simon C, Lowy R. Teenage childbearing. An adaptive strategy for the socioeconomically disadvantage or a strategy for adapting to socioeconomic disadvantage? Arch Pediatr Adolesc Med 1995; 149:912-5.

14.Rocha JSY, Simões BJG, Guedes GLM. Assistência hospitalar como indicador da desigualdade social. Rev Saúde Pública 1997; 31:479-87.

15. Townsend P, Davidson N. Inequalities in health: the Black report. $1^{\text {st }}$ ed. London: Penguin Books; 1982.

16.Singer P, Oliveira EM, Luchesi MA, Santos JLF, Nigro S. Demanda por alimentos na área metropolitana de Salvador. $1^{\mathrm{a}}$ ed. São Paulo: CEBRAP/Brasiliense; 1976. (Cad. CEBRAP, 23).

17. Organização Mundial da Saúde. Classificação Internacional de Doenças-CID. 9a revisão. Porto Alegre: Sagra/DC Luzzatto; 1975. p. 129-36.
18. Prado LV. Gravidez não planejada. Adolescência $1996 ; 45: 23-4$

19. Costa CO, Santos CAT, Nascimento Sobrinho CL, et al. Childbirth and live newborns of adolescent and young adult mothers in the municipality of Feira de Santana, Bahia State, Brazil, 1998. Cad Saúde Pública 2002; 18:715-22.

20.Martin JA, Park MM, Sutton PD. Births: preliminary data for 2001. Natl Vital Stat Rep 2002; 50:1-20.

21.Guijarro S, Naranjo J, Padilla M, Gutierez R, Lammers C, Blum RW. Family risk factors associated with adolescent pregnancy: study of a group of adolescent girls and their families in Ecuador. $J$ Adolesc Health 1999; 25:166-72.

22.Yazlle MEHD, Rocha JSY, Mendes MC, Patta MC, Marcolin AC, Azevedo GD. Incidência de cesáreas segundo fonte de financiamento da assistência ao parto. Rev Saúde Pública 2001; 35:202-6.

23.Blum RW, Geer L, Hutton L, et al. The Minnesota adolescent health survey. Implications for physicians. Minn Med 1988, 71:143-5, 149.

24.Upchurch DM, McCarthy J. The timing of a first birth and high school completion. Am Sociol Rev 1990; 55:224-34.

25.Bozkaya H, Mocan H, Usluca H, Beser E, Gumustekin D. A retrospective analysis of adolescent pregnancies. Gynecol Obstet Invest 1996; 42:146-50.

26.Gentile FP, Noronha Filho G, Cunha AA. Associação entre a remuneração da assistência ao parto e a prevalência de cesariana em maternidades do Rio de Janeiro: uma revisão da hipótese de Carlos Gentile de Mello. Cad Saúde Publica 1997; 13:221-6.

27.Kumbi S, Isehak A. Obstetric outcome of teenage pregnancy in northwestern Ethiopia. East Afr Med J 1999; 76:138-40.

28.Wasunna A, Mohammed K. Morbidity and outcome of low birthweight babies of adolescent mothers at Kenyatta National Hospital, Nairobi. East Afr Med J 2002; 79:539-42.

29.Balaka B, Baeta S, Agbere AD, Boko K, Kessie K, Assimadi K. Risk factors associated with prematurity at the University Hospital of Lome, Togo. Bull Soc Pathol Exot 2002; 95:280-3. 